\title{
Factores personales de la resiliencia y desempeño académico de escolares en un contexto de vulnerabilidad
}

\author{
Personal factors of resilience and academic performance of \\ schoolchildren in a context of vulnerability
}

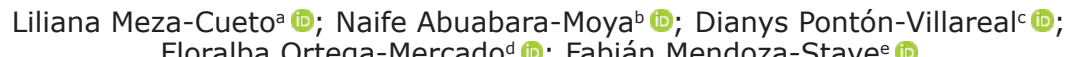
Floralba Ortega-Mercado ${ }^{d}$ (i); Fabián Mendoza-Stave ${ }^{\mathrm{e}}$ (i)

Recibido: 08-03-2020; Aceptado: 17-05-2020

\section{RESUMEN}

El presente estudio tuvo como objetivo establecer la relación entre los factores personales de la Resiliencia y el Desempeño Académico en el área de Lengua Castellana, determinando, además, diferencias según la Edad, Sexo y Nivel Socioeconómico. En cuanto al método, se trata de un estudio cuantitativo con alcance descriptivo-comparativo y un diseño no experimental de corte transversal, el cual empleó una muestra de 245 escolares colombianos en contexto de vulnerabilidad, con edades entre 10 y 18 años, seleccionados mediante muestreo probabilístico estratificado con asignación proporcional. Los instrumentos empleados fueron el inventario de factores personales de la Resiliencia y la Base de Datos del Desempeño Académico. Resultados: Los datos se analizaron mediante el coeficiente Pearson y la prueba ANOVA, y los resultados indicaron correlación entre el Desempeño Académico y la Resiliencia Global, en particular con los factores Empatía y Humor. Además, se determinó que no existen diferencias estadísticamente significativas entre los factores personales de la Resiliencia y el Sexo, Nivel Socioeconómico y la Edad, pero sí se muestran diferencias en el Desempeño Académico según el Sexo y el Nivel Socioeconómico. En cuanto al Desempeño Académico, no se encontraron diferencias según la variable Edad.

Palabras clave: Resiliencia; Desempeño Académico; Escolares; Vulnerabilidad

\section{ABSTRACT}

The objective of this study was to establish the relationship between personal factors of Resilience and Academic Performance in the area of the Spanish Language, also determining differences

aCorporación Universitaria del Caribe- CECAR, Programa Psicología. Sincelejo, Colombia. liliana.mezac@cecar.edu.co

bUniversidad de Cartagena, Facultad de Enfermería, Cartagena, Colombia. Secretaría de Educación de Bolívar, Gobernación de Bolívar, Mompox, Colombia. naifeabuabaramoya80@gmail.com

'Secretaría de Educación de Bolívar, Gobernación de Bolívar. Mompox, Colombia. alejipo@live.com

dUniversidad Nacional Abierta y a Distancia CCAV Corozal, Escuela de Ciencias Sociales, Artes y Humanidades. Corozal, Colombia. floralba. Ortega@unad.edu.co

eUniversidad Nacional Abierta y a Distancia CCAV Corozal, Dirección UNAD. Corozal, Colombia. fabian.mendoza@unad.edu.co 
according to Age, Sex and Socioeconomic Level. Regarding the method, it is a quantitative study with a descriptive-comparative scope and a non-experimental cross-sectional design, which used a sample of 245 Colombian schoolchildren in a context of vulnerability, aged between 10 and 18 years, selected by stratified probability sampling with proportional allocation.

The instruments used were the inventory of personal factors of Resilience and the Database of Academic Performance. Results: The data were analyzed using the Pearson coefficient and the ANOVA test, and the results indicated a correlation between Academic Performance and Global Resilience, particularly with the Empathy and Humor factors. In addition, it was determined that there are no statistically significant differences between personal factors of Resilience and Sex, Socioeconomic Level and Age, but differences in Academic Performance are shown according to Sex and Socioeconomic Level. Regarding Academic Performance, no differences were found according to the Age variable.

Keywords: Resilience; Academic Performance; Schoolchildren; Vulnerability

\section{INTRODUCCIÓN}

Colombia es uno de los países de América Latina con mayor desigualdad, siendo superado solo por Guatemala y México. Esto se evidencia en las brechas existentes entre regiones en materia de salud, educación, ingresos y acceso a oportunidades, siendo las poblaciones rurales las más afectadas. Así se confirma en un informe emitido por el Centro Latinoamericano de Desarrollo Rural - Rimisp (2017). Según algunos estudios, en Colombia son evidentes las condiciones de pobreza y exclusión, siendo estas más prevalentes en las Regiones Caribe y del Pacífico, en las que abundan también condiciones de vulnerabilidad y rezago (Galvis-Aponte y Alba-Fajardo, 2016). Estos mismos autores, afirman que en la Región Caribe colombiana un 68,7\% de los municipios se encuentra en situación de "rezago", es decir que se han quedado atrás sin avanzar.

En la Región Caribe se sitúa el Departamento de Bolívar, el cual muestra tasas preocupantes de pobreza, así como Nivel es de hambre por encima del promedio nacional $(7 \%)$, siendo los niños los más afectados; al menos 10 de cada 100 niños presentan peso insuficiente (Espinosa, Arrieta y Toro, 2007). Según el Banco Interamericano de Desarrollo-BID (2014), los niños son altamente vulnerables a la pobreza, dado que esta constituye un riesgo para su óptimo desarrollo a lo largo del tiempo, en tanto se traduce en factores como alimentación insuficiente, falta de higiene y condiciones familiares inadecuadas.

Uno de los municipios más vulnerables es Talaigua Nuevo, ubicado en la zona de la "depresión momposina" del Departamento de Bolívar, el cual, según Alcaldía municipal de Alcaldía Municipal de Talaigua Nuevo (2016), presenta Nivel es críticos de pobreza, hacinamiento poblacional y poco acceso al sistema de salud y hospitalario. Se conoce, además, que el municipio es receptor de víctimas del conflicto armado, estimando que se han recibido, al menos, 1.306 víctimas desplazadas (Turizo, 2018).

Ahora bien, no se puede pasar por Alto que este municipio ha sufrido también las consecuencias de la ola invernal, registrándose 9.300 personas afectadas (OCHOA, Colombia, 2010), cifra considerable si se tiene en cuenta que, según el último reporte del Departamento Nacional de Estadística-DANE, esta población cuenta con 11.167 habitantes (Alcaldía Municipal de Talaigua Nuevo, 2016). De esto, se conoce que los niños y adolescentes fueron la población más afectada, debido a que se generaron problemas de salud, como enfermedades diarreicas y respiratorias (Contreras, 2015). De hecho, este tipo de enfermedades son la primera y segunda causa de morbilidad y mortalidad infantil en el municipio (Alcaldía Municipal de Talaigua Nuevo, 2016).). 
Meza-Cueto et al - Factores personales de resiliencia y desempeño académico en un contexto de vulnerabilidad

De acuerdo con lo anterior, la Comisión Económica para América Latina y el Caribe-CEPAL (2017), argumenta que, frente a una población mayor de 18 años, los niños y niñas resultan ser más vulnerables ante los desastres, y experimentan sus efectos en Altos grados y durante un mayor tiempo.

Una investigación realizada por Bravo, Bustamante, Herazo, Godín y Meléndez (2016) arrojó al estudiar una muestra de niños y adolescentes expuestos a inundaciones a causa del fenómeno de "La Niña" en Colombia, que estos no tienen suficientes defensas para enfrentar este tipo de situaciones y que, por ende, son afectados a nivel emocional y psicosocial. Sin embargo, Meza y Palacio (2017) concluyeron que un grupo de niños y adolescentes residentes en municipios de la Región Caribe colombiana con inundaciones recurrentes, se encontraban adecuadamente adaptados en el ámbito personal, escolar y social, lo cual fue atribuido a características intrínsecas de los sujetos que les permitieron tener un comportamiento adaptativo ante la situación a lo largo del tiempo. Esto puede tornarse paradójico si se tienen en cuenta las condiciones de vulnerabilidad descritas; no obstante, estas circunstancias extremas en algunos casos, no impiden que el ser humano logre un desarrollo sano y positivo (Werner y Smith, 1992). Esto tiene sentido, si se observa que los Estudiantes de Secundaria de la Institución Educativa de Talaigua Nuevo, muestran progresos significativos en su Desempeño Académico, según el índice sintético de calidad educativa (Ministerio de Educación Nacional-MEN, 2018).

Lo anterior podría ser explicado a partir de la Resiliencia, la cual supone una capacidad del ser humano para desenvolverse de manera exitosa, tanto física como psicológicamente, a pesar de afrontar contextos difíciles que ponen en riesgo su integridad (Puerta y Vásquez, 2012; Quispe y Mendoza, 2017). Desde esta perspectiva, una persona es resiliente si luego de haber estado expuesta a situaciones adversas continúa dando resultados positivos (Galvis-Aponte y AlbaFajardo, 2016).

Diversos estudios indican una relación entre el Desempeño Académico y la Resiliencia (Gaxiola y González, 2020; Santiago, Vergel y Gallardo, 2020). Una investigación realizada por Arias (2013), arrojó al estudiar una muestra de escolares ecuatorianos de los grados $6^{\circ}, 7^{\circ}, 8^{\circ}, 9^{\circ}$ y $10^{\circ}$ que, a mayores Nivel es de Resiliencia mayor Desempeño Académico, hallazgo que es coherente con un estudio realizado por Gallesi y Matalinares (2012) en Estudiantes peruanos de Primaria. Del mismo modo, Gaxiola, González, Contreras y Gaxiola (2012) encontraron al estudiar adolescentes con disposiciones resilientes y no resilientes, que en el grupo resiliente las variables se relacionaron positivamente con el Desempeño Académico.

Una investigación realizada en Colombia con Estudiantes universitarios, arrojó que existe una relación estadísticamente significativa entre los Factores Resilientes y el Desempeño Académico (Peralta, Ramírez y Castaño, 2006). En este mismo sentido, un estudio realizado por Álvarez y Cáceres (2010), indica una relación Muy Baja entre Rendimiento Académico y Resiliencia, así como la existencia de diferencias entre hombres y mujeres en estas variables, siendo los sujetos masculinos menos resilientes que los femeninos (Aguirre, 2004; Matalinares et al., 2011), aunque no se encontraron diferencias según la Edad. En esta misma línea, los resultados de un estudio realizado por Tuñón y Poy (2016) muestran diferencias en el rendimiento escolar según el Sexo, en Estudiantes argentinos de Primaria y Secundaria, siendo las mujeres quienes obtuvieron un mayor rendimiento en la asignatura de Lengua.

Con relación al Desempeño Académico, al investigar una muestra representativa de Estudiantes mexicanos de Secundaria, Chaparro, González y Caso (2016), encontraron que aquellos Estudiantes con mayor rendimiento académico, tenían también un Alto Nivel Socioeconómico, dado que estos cuentan con recursos básicos y necesarios para sus actividades escolares. Otros estudios realizados con universitarios colombianos arrojaron que a menor Edad y Estrato Social mejor Desempeño Académico, dado que el ingreso a la escuela se percibe como una oportunidad para salir de la pobreza (Brito \& Palacio, 2016). 
Actualmente, el concepto de Resiliencia ha sido abordado desde la psicología desde diversas perspectivas teóricas (Werner y Smith, 1992; Bowlby, 1998; Kotliarenco, Cáceres \& Fontecilla, 1997; Villalta, 2010; Vanistendael, 2005; Salgado, 2005).

Según Salgado (2005), la Resiliencia se manifiesta a través de factores personales, definidos como aquellas capacidades y aptitudes que les permiten a las personas desarrollar estilos y estrategias de afrontamiento a partir del cultivo de los recursos internos con los que cuentan, para hacer frente a los problemas y situaciones difíciles de la vida diaria. Para esta autora, los cinco factores personales de la Resiliencia a desarrollar en la infancia son: Autoestima, Empatía, Autonomía, Humor y Creatividad, sobre los cuales se conceptualiza a continuación:

Autoestima: Es la capacidad para valorarse de manera positiva.

Empatía: Interacción basada en la adquisición premeditada del punto de vista del otro. Destreza y disposición para comunicarse de manera abierta y fluida con los otros, a pesar del ambiente, con interacción basada en la comprensión y reciprocidad con los valores y la situación de los/as demás.

Autonomía: Sentido de identidad propia y competencia para tomar decisiones y actuar independientemente y autorregular sus emociones; además, incluye reconocerse, cuidar de sí y valorar a los demás.

Humor: Es un recurso que permite reducir Nivel es de estrés, desasosiego y demás emociones negativas o percepción negativa de las emociones. A través del sarcasmo, de la caricaturización y lo disparatado, además ayuda a los individuos a superar las dificultades provocadas por las situaciones conflictivas de manera aceptable.

Creatividad: Se conoce como la capacidad de inventar nuevas propuestas a partir de aquello que se cree no tiene ninguna utilidad. También comprende la destreza para pensar de modo flexible, posibilitando dar soluciones nuevas ante problemas de distinta índole y la desesperanza. En los niños esta capacidad se manifiesta a través del juego (Wolin y Wolin, 1993).

Con relación al Desempeño Académico, es definido por Jiménez (2000) como el rango de conocimientos alcanzados en una asignatura o área, acorde con la Edad y el Nivel Cognitivo de Desarrollo. Por su parte, Montero, Villalobos y Valverde (2007) amplían esta visión conceptualizándolo como el conjunto de diversos elementos que se relacionan con los logros académicos, en el cual se conjugan factores psicológicos, sociales, demográficos, didácticos, y económicos; entre ellos, elementos tan diversos como la estimulación, la preocupación, la Autoestima, la apreciación del clima académico, el maestro, el sentido de vida y otros (Pérez y Londoño, 2015).

En este sentido, la legislación colombiana fija la valoración del Desempeño Académico a través del Decreto 1290 del 16 de abril de 2009. Específicamente, el Artículo 5 establece la escala de valoración nacional; sin embargo, advierte que las instituciones son autónomas al asignar la valoración para cada desempeño. No obstante, la escala se establece para facilitar el traslado de los Estudiantes cuando haya necesidad de cambiar de establecimiento educativo; de esta manera se deberá indicar su equivalencia con la escala de valoración nacional: 1) Desempeño Superior, 2) Desempeño Alto, 3) Desempeño Básico, 4) Desempeño Bajo. La calificación Superior, se entiende como excelencia en el alcance de todos los objetivos propuestos, teniendo en cuenta además de los procesos cognitivos, la formación integral del individuo, el desempeño Alto; y propone el alcance de las metas propuestas.

Teniendo en cuenta los elementos propuestos, la presente investigación buscó establecer la relación existente entre los factores personales de la Resiliencia y el Desempeño Académico en el área de Lengua Castellana, en Estudiantes de Básica Secundaria en situación de vulnerabilidad. 
Meza-Cueto et al - Factores personales de resiliencia y desempeño académico en un contexto de vulnerabilidad

Además, establecer la existencia de diferencias significativas entre los factores personales de la Resiliencia y el Desempeño Académico, con el Sexo, la Edad y el Nivel Socioeconómico. Todo esto, considerando el impacto de este tipo de estudios a corto y mediano plazo, dado que, de comprobarse la hipótesis respecto a la relación entre Resiliencia y Desempeño Académico, así como las diferencias según las variables sociodemográficas, podrían promoverse planes y programas escolares que fortalezcan las variables asociadas al rendimiento escolar y, de esta manera, lograr aumentar los indicadores de calidad educativa en la región y el país, dado que el estudio podría servir como una referencia nacional e incluso internacional. Del mismo modo, a través de este estudio, se impulsarían otras investigaciones que consideren variables de interés en el problema de investigación planteado, contribuyendo al abordaje de diversos problemas asociados al aprendizaje escolar.

\section{MÉTODO}

Enfoque, Alcance y Diseño. El enfoque de la presente investigación es cuantitativo, de tipo no experimental correlacional.

Población y Muestra. Mediante un muestreo probabilístico estratificado con asignación proporcional, se conformó una muestra de 245 Estudiantes de Secundaria matriculados en la Institución Educativa de Talaigua Nuevo ubicada en el Nivel Socioeconómico 1, de carácter estatal y en donde laboran algunos de los investigadores como docentes. Del total de la muestra, 122 son mujeres y 123 son hombres, sus Edades oscilan entre los 10 y los 18 años, de los cuales 23 se encuentran en la etapa de la niñez y 222 en la etapa de la adolescencia, estratificados socioeconómicamente así: 169 el Nivel 1 y 76 en el Nivel 2.

Consideraciones Éticas. Se solicitó autorización a los padres de familia mediante el diligenciamiento del consentimiento informado, de igual manera se trataron los datos bajo confidencialidad y el principio de ética en la investigación científica contemplado en la Resolución No. 8430 de 1993, específicamente el Capítulo III (De las investigaciones en menores de edad o discapacitados).

\section{Instrumentos}

Inventario de Factores Personales de la Resiliencia (Salgado, 2005). Este instrumento evalúa cinco factores personales de la Resiliencia: Autoestima, Empatía, Autonomía, Humor y Creatividad, y arroja un índice global de Resiliencia. Está compuesto por 48 ítems, de opción dicotómica: "si o no". El instrumento se encuentra validado en Colombia (Rodríguez, Guzmán y Yela, 2019).

Base de Datos de Desempeño Académico. Corresponde a la recopilación y análisis del reporte del promedio de calificaciones de los Estudiantes de Secundaria en el área de Lengua Castellana durante el año 2018. Esto se hace para determinar el porcentaje de Estudiantes en cada Nivel de rendimiento y así poder confrontar esos datos con la información del inventario de factores personales de la Resiliencia.

Análisis Estadístico. Los datos fueron analizados mediante el programa estadístico SPSS, empleando estadísticos descriptivos, R-Pearson para correlaciones y ANOVA para comparar grupos.

Procedimiento. En primera instancia, se procedió a solicitar la autorización de los padres de familia de los sujetos para su participación en el estudio. En segundo lugar, se aplicó el instrumento Inventario de Factores Personales de la Resiliencia y se procedió a la consolidación de la Base de Datos del Desempeño Académico del Área de Lengua española de los Estudiantes 
durante tres periodos académicos. Finalmente, se construyó una Base de Datos y se procesó la información utilizando el paquete estadístico SPSS (v. 24), derivando los resultados descriptivos, correlaciones y análisis de varianza.

\section{RESULTADOS}

Resultados Descriptivos de Factores Personales de la Resiliencia y Desempeño Académico. En cuanto a la Autoestima, se observa en la Tabla 1, que 184 Estudiantes, correspondientes al $75 \%$ de la muestra, poseen un Nivel Medio en la variable Autoestima lo que indica que se encuentran en el rango de normalidad. De la manera siguiente, el $22.4 \%$ de los Estudiantes poseen baja Autoestima, lo cual se refleja en un autoconcepto reducido.

Tabla 1. Factor Autoestima.

\begin{tabular}{cccccc}
\hline & & Frecuencia & Porcentaje & Porcentaje Válido & Porcentaje Acumulado \\
\hline \multirow{3}{*}{ Válido } & Bajo & 55 & 22,4 & 22,4 & 22,4 \\
& Medio & 184 & 75,1 & 75,1 & 97,6 \\
& Alto & 6 & 2,4 & 2,4 & 100,0 \\
& Total & 245 & 100,0 & 100,0 & \\
\hline
\end{tabular}

Fuente: Elaboración Propia.

La Tabla 2 muestra que el 74,3\% de los Estudiantes obtuvo un Nivel Medio de Empatía y el $3,3 \%$ un Nivel Alto, lo que indica que la mayoría posee algunas habilidades para experimentar y provocar simpatía, afecto y comprensión por las dificultades y necesidades de los otros y las suyas propias. Entre tanto, la Categoría Bajo promedia un 22,4\% de los Estudiantes, los cuales no alcanzan niveles significativos de Empatía, reflejando que no se identifican o encontrarán dificultades para identificarse con los demás.

Tabla 2. Factor Empatía.

\begin{tabular}{cccccc}
\hline & & Frecuencia & Porcentaje & Porcentaje válido & Porcentaje acumulado \\
\hline & Bajo & 55 & 22,4 & 22,4 & 22,4 \\
Válido & Medio & 182 & 74,3 & 74,3 & 96,7 \\
& Alto & 8 & 3,3 & 3,3 & 100,0 \\
& Total & 245 & 100,0 & 100,0 & \\
\hline
\end{tabular}

Fuente: Elaboración Propia

En la Tabla 3, se observa que respecto a la variable Autonomía los Estudiantes obtuvieron un Alto porcentaje en el Nivel Medio, marcando un 79,2\%, y Alto 4,9\%, de donde, se puede afirmar que tanto niños como adolescentes poseen capacidades para decidir sobre sus propias acciones, de acuerdo con sus necesidades. Sin embargo, no se puede desconocer que un $15,9 \%$ obtuvo un promedio Bajo en este factor, lo que indica que no son independientes y necesitan contar con apoyo para disponer de su propia vida.

Tabla 3. Factor Autonomía.

\begin{tabular}{cccccc}
\hline & & Frecuencia & Porcentaje & Porcentaje Válido & Porcentaje Acumulado \\
\hline \multirow{3}{*}{ Válido } & Bajo & 39 & 15,9 & 15,9 & 15,9 \\
& Medio & 194 & 79,2 & 79,2 & 95,1 \\
& Alto & 12 & 4,9 & 4,9 & 100,0 \\
& Total & 245 & 100,0 & 100,0 & \\
\hline
\end{tabular}

Fuente: Elaboración Propia 
Se evidencia la Tabla 4, con relación a la variable Humor, que un $62 \%$ de Estudiantes obtuvo puntuación Media y $3,7 \%$ se sitúa en el Nivel Alto, lo quiere decir que tienen disposición del espíritu a la alegría, para encontrar lo positivo en los momentos adversos; en tanto un 34,3\% presenta un Nivel Bajo en este factor. Esto puede relacionarse con la falta de vínculos positivos entre las situaciones y la forma como estos estudiantes las asumen, así como de recursos para "activar procesos resilientes" mediante la variable Humor.

Tabla 4. Factor Humor.

\begin{tabular}{cccccc}
\hline & & Frecuencia & Porcentaje & Porcentaje válido & Porcentaje acumulado \\
\hline \multirow{3}{*}{ Válido } & Bajo & 84 & 34,3 & 34,3 & 34,3 \\
& Medio & 152 & 62,0 & 62,0 & 96,3 \\
& Alto & 9 & 3,7 & 3,7 & 100,0 \\
& Total & 245 & 100,0 & 100,0 & \\
\hline
\end{tabular}

Fuente: Elaboración Propia

La Tabla 5 indica que, con relación a la variable Creatividad, se obtuvo un 68,2\% de Estudiantes en el Nivel Medio, $24,9 \%$ en el Nivel Alto y 6,9\% en el Nivel Bajo, lo cual es indicador de que una gran proporción de sujetos posee capacidades para transformar su entorno, generar ideas o propuestas innovadoras, dar soluciones novedosas, demostrando destrezas para el pensamiento creativo.

Tabla 5. Factor Creatividad.

\begin{tabular}{cccccc}
\hline & & Frecuencia & Porcentaje & Porcentaje Válido & Porcentaje Acumulado \\
\hline \multirow{4}{*}{ Válido } & Bajo & 17 & 6,9 & 6,9 & 6,9 \\
& Medio & 167 & 68,2 & 68,2 & 75,1 \\
& Alto & 61 & 24,9 & 24,9 & 100,0 \\
& Total & 245 & 100,0 & 100,0 & \\
\hline
\end{tabular}

Fuente: Elaboración Propia

En la Tabla 6 , se observa que un $69,4 \%$ de los Estudiantes obtuvo un puntaje Promedio de Resiliencia, el cual corresponde a 170 Estudiantes, lo que indica que la mayoría de ellos poseen elevado grado de capacidades resilientes $(69,4+2,4)$ en cierto grado (Bajo + Medio; 87,8\%). De otro lado, 24 Estudiantes, es decir 9,0\%, presentan un Nivel Muy Bajo de Resiliencia y 45 Estudiantes, correspondientes al 18,4\%, un Nivel Bajo, lo que significa que es poca la población que posee Niveles Reducidos de Resiliencia (Muy Bajo + Bajo; 28,2\%). Asimismo, es de destacar que 6 Estudiantes, correspondientes al 2,4\% muestran Alto Nivel de Resiliencia. En conjunto, estos resultados muestran que los escolares se encuentran en el camino hacia la construcción de resultados positivos a partir de la Resiliencia.

Tabla 6. Resultado Resiliencia Global.

\begin{tabular}{cccccc}
\hline & & Frecuencia & Porcentaje & Porcentaje Válido & Porcentaje Acumulado \\
\hline & Muy Bajo & 24 & 9,8 & 9,8 & 9,8 \\
Válido & Bajo & 45 & 18,4 & 18,4 & 28,2 \\
& Medio & 170 & 69,4 & 69,4 & 97,6 \\
& Alto & 6 & 2,4 & 2,4 & 100,0 \\
& Total & 245 & 100,0 & 100,0 & \\
\hline
\end{tabular}

Fuente: Elaboración Propia

En lo referente a la variable Desempeño Académico, en la Tabla 7 se observa que el porcentaje Más Alto se encuentra en el desempeño Básico con un $41,6 \%$, seguido del desempeño Alto con un 33,1\%, mientras que los porcentajes Más Bajos los presentan los desempeños Bajo y Superior 
con $16,3 \%$ y $9,0 \%$, respectivamente. De acuerdo con esto, se evidencia que los Estudiantes alcanzan los objetivos académicos propuestos para ellos.

Tabla 7. Resultados Descriptivos: Desempeño Académico.

\begin{tabular}{cccccc}
\hline & & Frecuencia & Porcentaje & Porcentaje Válido & Porcentaje Acumulado \\
\hline & Bajo & 40 & 16,3 & 16,3 & 16,3 \\
Válido & Básico & 102 & 41,6 & 41,6 & 58,0 \\
& Alto & 81 & 33,1 & 33,1 & 91,0 \\
& Superior & 22 & 9,0 & 9,0 & 100,0 \\
& Total & 245 & 100,0 & 100,0 & \\
\hline
\end{tabular}

Fuente: Elaboración Propia

Correlaciones entre Factores Personales de la Resiliencia y el Desempeño Académico. Teniendo en cuenta que la muestra fue mayor a 50, se aplicó la prueba de normalidad KolmogorovSmirnov hallando una distribución normal debido a que el nivel de significancia para las variables de estudio fue mayor a 0,05, tal como se observa en la tabla 8 . Por tanto, se procedió a aplicar la prueba estadística R-Pearson como prueba paramétrica.

Tabla 8. Prueba de normalidad Kolmogorov-Smirnov.

\begin{tabular}{cccc}
\hline & \multicolumn{3}{c}{ Kolmogorov-Smirnov } \\
\cline { 2 - 4 } & Estadístico & gl & Sig. \\
\hline Desempeño académico &, 085 & 245 & 0,071 \\
Autoestima &, 219 & 245 & 0,276 \\
Empatía &, 232 & 245 & 0,234 \\
Autonomía &, 164 & 245 & 0,198 \\
Humor &, 171 & 245 & 0,143 \\
Creatividad &, 143 & 245 & 0,165 \\
Resiliencia global &, 230 & 245 & 0,165 \\
\hline
\end{tabular}

Fuente: Elaboración propia

En la Tabla 9, se observa que hay una correlación significativa entre Desempeño Académico y la Resiliencia global, $(r=0,168, p<0,09)$ Asimismo, el Desempeño Académico presenta una correlación significativa con respecto a las dimensiones Empatía $(r=0,135 ; p<0,03)$ y Humor $(r=0,134 ; p<0,03)$.

Tabla 9. Correlación entre Desempeño Académico y Factores Personales de la Resiliencia.

\begin{tabular}{lccccccc}
\hline & & Autoestima & Empatía Autonomía Humor & Creatividad Resiliencia global \\
\hline \multirow{2}{*}{$\begin{array}{c}\text { Desempeño } \\
\text { Académico }\end{array}$} & Correlación de Pearson &, 104 &, $135^{*}$ &, 101 &, $134^{*}$ &,- 074 &, $168^{* *}$ \\
& Sig. (bilateral) &, 103 &, 034 &, 115 &, 036 &, 251 &, 009 \\
\hline
\end{tabular}

** La correlación es significativa al Nivel 0,01 (bilateral); *La correlación es significante al Nivel 0,05 (bilateral)

Fuente: Elaboración Propia

Diferencias entre Factores Personales de la Resiliencia y Desempeño Académico según Sexo, Edad y Nivel Socioeconómico. Tal como se muestra en la Tabla 10, existen diferencias significativas entre hombres y mujeres respecto a su Desempeño Académico, $(F=26.132$, $\mathrm{P}=0.000$ ); se encuentra que las mujeres obtuvieron una media Más Alta que los hombres. Del mismo modo, se observan diferencias significativas entre el Nivel Socioeconómico y el Desempeño 
Meza-Cueto et al - Factores personales de resiliencia y desempeño académico en un contexto de vulnerabilidad

Académico ( $F=7.36, P=0.007)$; los sujetos de Estrato $2(M=2,57)$ tuvieron un Desempeño Académico Más Alto que los de Estrato $1(M=2,25)$. Con relación a la Edad, no se encontraron diferencias en el Desempeño Académico.

Tabla 10. Estudio de Significatividad entre Desempeño Académico y las Variables Sexo, Edad y Nivel Socioeconómico.

\begin{tabular}{ccccccc}
\hline \multirow{3}{*}{ Sexo } & & N & Media & Desviación Estándar & F & Sig. Anova \\
& Masculino & 123 & 2,08 &, 785 & 26,132 &, 000 \\
& Femenino & 122 & 2,61 &, 847 & & \\
\multirow{5}{*}{ Edad } & Total & 245 & 2,35 &, 858 &, 578 &, 448 \\
& Niños & 22 & 2,22 &, 850 & & \\
& Adolescentes & 222 & 2,36 &, 859 & & \\
\multirow{2}{*}{ Estrato } & Total & 245 & 2,35 &, 858 & 7,36 & \\
& 1 & 169 & 2,25 &, 822 & & \\
& 2 & 76 & 2,57 &, 899 & & \\
& Total & 245 & 2,35 &, 858 & & \\
\hline
\end{tabular}

Fuente: Elaboración propia

No obstante, no se encontraron diferencias significativas entre los factores personales de la Resiliencia y el Sexo, Edad y Nivel Socioeconómico.

\section{DISCUSIÓN}

El estudio pretendió establecer una relación entre la Resiliencia y el Desempeño Académico en una muestra de escolares en condición de vulnerabilidad, así como estudiar las diferencias en función de las variables Edad, Sexo y Nivel Socioeconómico. Los resultados obtenidos indicaron que existe una correlación significativa entre el Nivel de Resiliencia global y el Desempeño Académico, lo cual va en la misma línea de los hallazgos de estudios previos (Prado y Del Águila, 2003; Álvarez y Cáceres, 2010; Arias, 2013; Huaire, 2014; Quispe y Vera, 2017; Coppari, Barcelata, Bagnoli y Codas, 2018). En coherencia con esto, la Resiliencia propone un conglomerado de factores asociados al contexto y a la personalidad, que le permiten al sujeto enfrentarse a las situaciones adversas que se le presenten en la vida, ahora bien, estos factores aportan cualidades necesarias para el éxito educativo y familiar, así como para el desenvolvimiento positivo en la sociedad. (Grotberg, 1995; Kotliarenco, 2000; Murillo, 2003; Gallesi y Matalinares, 2012).

Algunos factores personales de la Resiliencia correlacionaron con el Desempeño Académico, como es el caso de la Empatía, lo cual es coherente con lo planteado por Gallesi y Matalinares (2012), quienes aportan que la Empatía correlaciona significativamente con el Desempeño Académico, dado que los sentimientos hacia los pares o semejantes generan comprensión y simpatía, lo que conducirá a mejoras significativas en todas las áreas. Esto puede obedecer a que las interacciones con los otros enriquecen la motivación hacia el logro de objetivos y propician un contexto de cooperativismo que sin lugar a dudas potenciará el Desempeño Académico. Del mismo modo, se encontró que el factor Humor está correlacionado con el Desempeño Académico, resultado que coincide con los hallazgos de Gallesi y Matalinares (2012).

Ahora bien, en el presente estudio no se encontró correlación entre Autoestima y Desempeño Académico, lo cual va en el mismo sentido de lo hallado en otros estudios (Aluicio y Revellino, 2011; Chilca, 2017, Morales y González, 2014). Sin embargo, difiere de lo arrojado en estudios realizados por Yapura (2015) y Laguna (2017) en los que los Estudiantes con Alta Autoestima presentaron 
Excelente Desempeño y los que presentaban Baja Autoestima, evidenciaron Bajo Rendimiento Académico. Asimismo, García y Musitu (1993) manifiestan que la Autoestima y el Desempeño Académico se influyen mutuamente, afirmando que es imprescindible tener una Autoestima Alta para conseguir buenos resultados académicos. Al respecto, Rabell (2012) explica que la Autoestima puede explicar la variabilidad en el rendimiento académico, pero no completamente, pues solo es una porción, dado que existen otras condiciones del entorno que pueden ser determinantes tales como los contextos académicos, personales y familiares que podrían estar asociados al desempeño de los Estudiantes y condicionando su rendimiento académico, hasta el punto de permitir o negar la posibilidad de tener Altas Calificaciones con Baja Autoestima.

Otra de las variables que no correlacionó con el Desempeño Académico fue la Autonomía, en contraposición a lo citado en algunos estudios los cuales plantean que a través de la Autonomía se potencia el aprendizaje y se obtienen aprendizajes significativos en el Aula (Franca, 2016; Paladines, 2016). Así pues, una posible solución a esta controversia estaría dada por el hecho de que la Autonomía estaría relacionada en el Desempeño Académico dependiendo del contexto dentro y fuera de la escuela, las prácticas de crianza y las personas que rodean el entorno del Estudiante. Sin embargo, las disimilitudes en estos hallazgos, invitan a continuar estudiando esta posible relación en muestras más representativas y en diversos contextos.

Con relación al factor Creatividad, en este estudio se encontró que no tiene correlación con el Desempeño Académico en el área de español, sin embargo, resulta paradójico que el factor Creatividad fue uno de los que obtuvo puntajes más Altos, lo que quiere decir que los Estudiantes en su gran mayoría han desarrollado esta competencia. En este mismo sentido, Alfonso (2017) afirma que la variable Creatividad no correlaciona de modo significativo con el rendimiento académico, pero es necesario potenciarla, por lo que plantea la necesidad de realizar propuestas de intervención educativa en la búsqueda de mejorar los resultados de los Estudiantes para que estos sean transformadores de sus entornos.

De otra parte, al intentar establecer diferencias entre los factores personales de la Resiliencia y las variables sociodemográficas, se encuentra que no existen diferencias significativas entre los factores personales de la Resiliencia y la variable Sexo, resultado que coincide con lo hallado por Prado y Del Águila (2003) quienes demostraron que no hay diferencias significativas en las puntuaciones totales de la Resiliencia respecto al Sexo. Esto obedece a que las situaciones conflictivas y hostiles de la vida incluyen a todos los seres humanos sin distinguir género, lo cual tiende a adiestrar a los sujetos para enfrentar expectativas contrarias a lo esperado, fortaleciendo por ende la Resiliencia sin importar el género.

Sin embargo, estos hallazgos discrepan con los de Gallesi y Matalinares (2012) quienes en su estudio encuentran que las mujeres obtienen mayores promedios de Resiliencia respecto a los hombres. Según estos autores, esto puede obedecer a que las habilidades para adaptarse a situaciones adversas en las mujeres son más eficaces, puesto que ellas no se preocupan por competir lo cual puede funcionar como un factor protector. En tal sentido, las discrepancias entre estos estudios sugieren la continuidad de investigaciones sobre estas diferencias.

Por otro lado, en lo que respecta a los factores personales de la Resiliencia y sus diferencias según la variable Edad, se encontró que no existen diferencias significativas, siendo consistente este hallazgo con el estudio realizado por Álvarez y Cáceres (2010) quienes no encontraron correlaciones significativas entre la Resiliencia y la Edad.

Con relación al Nivel Socioeconómico, se comprobó que no existen diferencias significativas con los factores personales de la Resiliencia, lo cual confirma los aportes de Grotberg (2006) en los que el Nivel Socioeconómico no está relacionado con la Resiliencia. Asimismo, Prado y Del Águila (2003) encontraron que en general no existen diferencias significativas en los valores totales entre la Resiliencia y el Estrato, esto significa que las clases sociales no son determinantes en la Resiliencia, lo cual también fue hallado en el estudio de Álvarez y Cáceres (2010). 
En lo que respecta a la variable Desempeño Académico, se encontró que las mujeres presentaron un Nivel Más Alto que los hombres, lo que coincide con los estudios previos (Di Gresia, 2002; Echavarri, Godoy y Olaz, 2007; Gallesi y Matalinares, 2012; Peralta, Ramírez y Castaño, 2006, Porto y Di Gresia, 2004, Tuñón y Poy, 2016). Esto se justifica, de acuerdo con estos autores, porque las mujeres parecen estar más motivadas para todo lo concerniente al trabajo académico lo cual les permite desarrollar habilidades que hacen que se destaquen en este ámbito.

En lo que compete a las diferencias en el Desempeño Académico según la Edad, en esta investigación no se hallaron diferencias significativas, lo cual es coherente con los resultados de la investigación realizada por Rojas, Arbeláez y Prieto (2016) en la que se concluye que la Edad no es determinante en el Desempeño Académico, dado que se encontró una correlación negativa muy baja entre ambas variables. Sin embargo, contrario a los resultados obtenidos en esta investigación, Brito y Palacio (2016) afirman que a menor Edad mejor Desempeño Académico, obedeciendo esto a ciertas características tales como el deseo de superarse, la motivación hacia el logro de metas y la esperanza de un mejor futuro; cualidades particulares de la población especifica del estudio realizado por estos autores. Tal como se observa, no existe un consenso en los hallazgos científicos respecto a las diferencias en el Desempeño Académico según la Edad, lo cual podría llevar a considerar características propias de cada contexto y del nivel de maduración de cada sujeto en particular según sus condiciones biológicas o socioculturales; sin embargo, esta sería una línea interesante para futuros estudios.

Ahora bien, los resultados mostraron diferencias significativas entre el Desempeño Académico y el Nivel Socioeconómico, dado que los Estudiantes pertenecientes al Estrato 1 presentaron un nivel de desempeño menor en comparación con los Estudiantes del Estrato 2. Este hallazgo, aunque difiere de algunos estudios previos (Peralta, Ramírez y Castaño, 2006; Guevara, Tovar y Jaramillo, 2013), es coherente con otras investigaciones realizadas (Cervini, 2002; Gil, 2013; Coschiza, Fernández, Redcozub, Nievas y Ruiz, 2016; Chaparro, González y Caso, 2016; Jadue, 1997; Suleman, Aslam, Hussain, Shakir y Khan, 2012; Tuñón y Poy, 2016). Una explicación a esas diferencias podría atribuirse a las condiciones de pobreza que inhabilitan el acceso a los recursos, materiales y tecnología, elementos que pueden resultar determinantes para el desarrollo de habilidades cognitivas en los Estudiantes (Chaparro, González y Caso, 2016; Gil, 2013; Moncayo, 2016).

Del presente estudio se resalta su pertinencia para la promoción de estrategias escolares orientadas al abordaje y fomento de la Resiliencia como factor asociado al rendimiento académico de los Estudiantes en contextos de vulnerabilidad, así como la construcción de estrategias, que, bajo un enfoque diferencial, potencien el rendimiento académico de los escolares. Como sugerencia, se plantea continuar estudiando estas correlaciones y diferencias según las características sociodemográficas en diversos contextos.

\section{Conflicto de intereses}

Los autores declaran no tener ningún conflicto de intereses.

\section{REFERENCIAS}

Alcaldía Municipal de Talaigua Nuevo. (2016). Plan de desarrollo económico, social, ambiental y de obras públicas 2016 - 2019. Talaigua más social y reconciliado. Talaigua Nuevo. http://talaiguanuevobolivar.micolombiadigital.gov.co/sites/talaiguanuevobolivar/content/ files/000041/2023 acuerdo-05-de-31-de-mayo-de-2016-pdm.pdf 
Alfonso, J. (2017). Correlación entre Creatividad, Estrategia de aprendizaje y rendimiento académico y plan de mejora para trabajar con niños de Secundaria [Tesis de maestría, Universidad internacional de la Rioja, España], https://reunir.unir.net/bitstream/handle/123456789/4549/ ALFONSO $\% 20$ MEDINA $\% 2$ C $\% 20 J 0 S E \% 20$ CRISANTO.pdf? sequence $=1$

Aluicio, A., y Revellino, M (2011). Relación entre autoeficacia, Autoestima, asertividad, y rendimiento académico, en Estudiantes que ingresaron a Terapia Ocupacional, el año 2010. Revista Chilena de Terapia Ocupacional, 11 (2), 1-15. https://tecnovet.uchile.cl/index.php/ RTO/article/view/17775

Álvarez, L., y Cacéres, L. (2010). Resiliencia, Rendimiento académico y variables sociodemográficas en Estudiantes universitarios de Bucaramanga (Colombia). Psicología Iberoamericana, 18(2), 37-46. http://www.redalyc.org/html/1339/133915921005/

Aguirre. A. (2004). Capacidad y factores asociados a la Resiliencia, en adolescentes del C.E. Mariscal Andrés Avelino Cáceres del Sector IV de Pamplona Alta San Juan de Miraflores 2002. [Trabajo de grado, Universidad Nacional Mayor de San Marcos, Perú] http://sisbib. unmsm.edu.pe/bibvirtualdata/tesis/salud/aguirre ga/T completo.PDF

Arias, F. M. (2013). La Resiliencia y el rendimiento académico que presentan los estudiantes de 6to, 7mo, 8vo, 9no y 10 mos cursos de la Escuela Ciudad de San Gabriel Quito - Ecuador [Tesis de pregrado, Universidad Central de Ecuador]. http://www.dspace.uce.edu.ec/ handle/25000/3414

Banco Interamericano de Desarrollo (2014). Programa Regional de Indicadores de Desarrollo Infantil (PRIDI). https://publications.iadb.org/es/publicacion/15178/programa-regional-deindicadores-de-desarrollo-infantil-pridi-marco-conceptual

Bowlby, J. (1998). El apego. Paidós.

Bravo, L., Bustamante, J., Herazo, M., Godin, R., y Meléndez, Y. (2016). Estado emocional de niños y adolescentes afectados por el invierno. Avances en Psicología: Revista de la Facultad de Psicología y Humanidades, 24(1) 73-78. http://revistas.unife.edu.pe/index.php/ avancesenpsicologia/article/view/145

Brito, I., y Palacio, J. (2016). Calidad de vida, Desempeño Académico y variables sociodemográficas en Estudiantes universitarios de Santa Marta - Colombia. Duazary, 13(2), 133 - 141. https:// doi.org/10.21676/2389783X.1719

Centro Latinoamericano para el Desarrollo Rural-Rimisp (2018). Pobreza y desigualdad, informe latinoamericano 2017. https://idl-bnc-idrc.dspacedirect.org/handle/10625/57430

CEPAL (Mayo de 2017). Niñez y desastres en América Latina y el Caribe. Desafíos, boletín de infancia y adolescencia, número 20. https://crm.cepal.org/civicrm/mailing/view?reset=1\&a $\underline{\mathrm{mp} \% 3 \mathrm{Bid}=159 \text { \# articulo central }}$

Contreras, R. (18 de Septiembre de 2015). Informe de personería municipal. Informe de damnificados del municipio de Talaigua Nuevo. Talaigua Nuevo.

Cervini, R. (2002). Desigualdades en el logro académico y reproducción cultural en Argentina: Un modelo de tres Nivel es. Revista Mexicana de Investigación Educativa, 7(16), 445-500. https://www.redalyc.org/articulo.oa?id=140/14001604

Coppari, N., Barcelata, B., Bagnoli, L., y Codas, G. (2018). Efectos de la Edad, el Sexo, el contexto cultural en la disposición resiliente de los adolescentes de Paraguay y México. Revista de Psicología Clínica con Niños y Adolescentes, 5(1), 16-22. http://dspace.umh.es/ bitstream/11000/4473/1/2-17-04.pdf 
Meza-Cueto et al - Factores personales de resiliencia y desempeño académico en un contexto de vulnerabilidad

Coschiza, C., Fernández, J., Redcozub, G., Nievas, M., y Ruiz, H. (2016). Características Socioeconómicas y Rendimiento Académico. El Caso de una Universidad Argentina. Revista Iberoamericana sobre Calidad, Eficacia y Cambio en Educación, 14(3), 51-76. https://doi. org/10.15366/reice2016.14.3.003

Chaparro, A. A., González, C. y Caso, J. (2016). Familia y rendimiento académico: configuración de perfiles estudiantiles en Secundaria. Revista Electrónica de Investigación Educativa, 18(1), 53-68. http://redie.uabc.mx/redie/article/view/774

Chilca, M. (2017). Autoestima, hábitos de estudio y rendimiento académico en Estudiantes universitarios. Propósitos y Representaciones, 5 (1), 71 - 127. https://dialnet.unirioja.es/ servlet/articulo?codigo $=5904759$

Di Gresia L., Porto A. y Ripani L. (2002). Rendimiento de Estudiantes de las universidades públicas argentinas. http://www.depeco.econo.unlp.edu.ar/doctrab/doc45.pdf

Echavarri, M., Godoy, J., y Olaz, F. (2007). Diferencias de género en habilidades cognitivas y rendimiento académico en Estudiantes universitarios. Universitas Psychologica, 6(2), 319329. https://revistas.javeriana.edu.co/index.php/revPsycho/article/view/120

Espinosa, A., Arrieta, J., y Toro, D. (2007). Bolívar y los Objetivos de Desarrollo del Milenio. Revista economía y Región, 28(1), 1-29. http://dx.doi.org/10.2139/ssrn.1033374

Franca, A. (2016). Autonomía, Autoestima, rendimiento académico [Trabajo de grado de maestría, Universidad de Sevilla, España]. https://idus.us.es/xmlui/handle/11441/45074https://idus. us.es/xmlui/handle/11441/45074

Gallesi, R., y Matalinares, M. (2012). Resiliencia y rendimiento académico en Estudiantes de $5^{\circ}$ y $6^{\circ}$ de Primaria. Revista de Investigación en psicología, 15(1). 181-201. http://dx.doi. org/10.15381/rinvp.v15i1.3677

Galvis-Aponte, L., y Alba-Fajardo, C. (2016). Dinámica de la pobreza en Colombia: vulnerabilidad, exclusión y mecanismos de escape. Documentos de trabajo sobre economía regional, 244, 1-42. http://www.banrep.gov.co/es/dtser-244

García, F. J., y Musitu, G. (1996). Rendimiento académico y Autoestima en el ciclo Superior de EGB. Revista de psicología de la educación, 4(11), 73 -87. http://repositori.uji.es/ xmlui/bitstream/handle/10234/168456/1993 Garcia\%20Bacete\%20y\%20Musitu Rendimiento\%20acad\%c3\%a9mico\%20y\%20Autoestima Rev\%20Psicologia\%20de\%20 la\%20Educacion $\% 281 \% 29$. pdf? sequence $=1$ \&isAllowed $=y$

Gaxiola, J., González, S. C., y Gaxiola, E. (2012). Predictores del rendimiento académico en adolescentes con disposiciones resilientes y no resilientes. Reivsta de psicología, 30(1), 47-74. http://www.scielo.org.pe/scielo.php?script=sci arttext\&pid=S0254-92472012000100003

Gaxiola, J., y González, S. (2020). Perceived Support, Resilience, Goals, and Self-Regulated Learning in High School Students. Revista electrónica de investigación educativa, 21, e08. http://dx.doi.org/10.24320/redie.2019.21.e08.1983

Gil, J. (2013). Medición del Nivel Socioeconómico familiar en el alumnado de Educación Primaria. Revista de Educación, 362, 298-322. https://dialnet.unirioja.es/servlet/ articulo?codigo $=4477746$

Grotberg, E. (1995). El Proyecto Internacional de Resiliencia: Promoviendo la Resiliencia en los Niños. Universidad de Wisconsin.

Grotberg, E. (2006). La Resiliencia en el mundo de hoy. Gedisa. 
Guevara, E., Tovar, S., y Jaramillo, R (Septiembre-Diciembre de 2013). Factores familiares y su relación con el rendimiento académico en Estudiantes de psicología. Revista Virtual Universidad Católica del Norte, 40, 122-140 http://www.redalyc.org/pdf/1942/194229200009.pdf

Huaire, E. (2014). Prevalencia de Resiliencia y Autoestima sobre el rendimiento escolar en Estudiantes de instituciones educativas de Ate Vitarte, Lima. Apunt. cienc. 04(02), 202-209. https://doi.org/10.18259/acs.2014023

Jadue, G. (1997). Factores ambientales que afectan el rendimiento escolar de los niños provenientes de familias de bajo Nivel Socioeconómico y Cultural. Estudios Pedagógicos, 23, 75-80.

Jiménez, M. (2000). Competencia social: Intervención preventiva en la escuela. Infancia y sociedad: revista de estudios, $24,21-48$. https://dialnet.unirioja.es/servlet/articulo?codigo $=4353980$

Kotliarenco, M. A. (2000). Actualizaciones en Resiliencia. UNLA.

Kotliarenco, M. A., Cáceres, I., \& Fontecilla, M. (julio de 1997). Estado de arte en Resiliencia. (O. M. Salud, Ed.) Paho. https://www.paho.org/Spanish/HPP/HPF/ADOL/Resil6x9.pdf

Laguna, N. M. (2017). La Autoestima como factor influyente del Desempeño Académico [Trabajo de grado maestría, Universidad del Tolima, Ibagué].http://repository.ut.edu. co/bitstream/001/2120/1/APROBADO\%20NIRZA\%20MARISOL\%20LAGUNA\% 20 PROA\%C3\%910S.pdf

Matalinares, M., Arenas, C., Yaringaño, J., Sotelo, L., Sotelo, N., Díaz, G., y otros. (2011). Factores personales de Resiliencia y autoconcepto en Estudiantes de Primaria de Lima Metropolitana. Revista de Investigaciones Psicológicas. 14(1), 187-207. https://doi.org/10.15381/rinvp. $\underline{\mathrm{v} 14 \mathrm{i} 1.2083}$

Meza, L., y Palacio, J. (2017). Funcionalidad familiar y apoyo social en niños, niñas y adolescentes afectados por el invierno, En A. Aguilar, A. Orozco \& M. Jiménez. Educación, Desarrollo y representaciones sociales: Estudios interdisciplinarios del Caribe. Ediciones Simón Bolívar. https://bonga.unisimon.edu.co/bitstream/handle/20.500.12442/2203/Cap 9 FuncionalidadFamiliar.pdf? sequence $=13$ \&isAllowed $=y$

Ministerio de Educación Nacional-MEN. (2018). Reporte de la excelencia. Índice Sintético de Calidad, Institución Educativa de Talaigua Nuevo. http://aprende.colombiaaprende.edu.co/ siemprediae/86438

Ministerio de Educación Nacional. (16 de abril de 2019). Artículo 5. Escala de Valoración Nacional. Decreto de Evaluación 1290.

Moncayo, M. A. (2016). Determinantes que influyen en el rendimiento académico: Un estudio aplicado para Colombia a partir de las pruebas ICFES-SABER 11. Universidad de la Salle. http:// repository.lasalle.edu.co/bitstream/handle/10185/20691/10091033 2016.pdf?sequence $=1$

Montero, E., Víllalobos, J., y Valverde, A. (2007). Factores institucionales, pedagógicos, Psicosociales y sociodemográficos, asociados al rendimiento académico en la universidad de Costa RIca: Un análisis multinivel. Revista electrónica de investigación y evaluación educativa. RELIEVE, 13(2), 215 -234. https://www.uv.es/RELIEVE/v13n2/RELIEVEv13n2 5.pdf

Morales, M., y González, A. (2014). Resiliencia-Autoestima-Bienestar psicológico y Capacidad intelectual de Estudiantes de cuarto medio de buen rendimiento de liceos vulnerables. Estudios pedagógicos (Valdivia), 40(1), 215-228. https://dx.doi.org/10.4067/S0718$\underline{07052014000100013}$ 
Meza-Cueto et al - Factores personales de resiliencia y desempeño académico en un contexto de vulnerabilidad

Murillo, J. (2003). La investigación sobre eficacia escolar en Iberoamerica Revisión internacional del estado del arte. Convenio Andrés Bello. https://www.researchgate.net/ publication/288604284 La investigacion sobre eficacia escolar en Iberoamerica Revision internacional del estado del arte

OCHOA, Colombia. (2010). Temporada de lluvias 2010, fenómeno de la niña. Informe de situación No 6. https://www.paho.org/disasters/index.php?option=com docman\&view=download\&category slug=colombia\&alias=1630-informe-ocha-colombiaola-invernal\&Itemid=1179\&lang=en

Osorio, J. (2017). Estrategias socio-pedagógicas basadas en la Resiliencia para fortalecer el rendimiento. UNAD. https://repository.unad.edu.co/bitstream/handle/10596/13936/ TESISResiliencia\%20jackeline.pdf?sequence $=1$

Paladines, R. (2016). Incidencia de la Autonomía educativa y el aprendizaje de los Estudiantes del colegio Juan Montalvo del cantón Manta. Dominio de las Ciencias, 2(4), 133-148. https:// dialnet.unirioja.es/servlet/articulo?codigo $=5761633$

Peralta, S., Ramírez, A., y Castaño, H. (2006). Factores resilientes asociados al rendimiento académico en Estudiantes pertenecientes a la universidad de Sucre. Psicología desde el caribe, 1(17), 196-219. http://rcientificas.uninorte.edu.co/index.php/psicologia/article/ viewFile/2054/9366

Pérez, D. C., y Londoño, D. A. (2015). La influencia de la familia en el Desempeño Académico de los y las adolescentes del grado sexto en tres instituciones de Antioquia. Virtual de ciencias sociales y humanas psicoespacios, 9(15), 215-233. https://dialnet.unirioja.es/descarga/ articulo/5317692.pdf

Porto, A. y Di Gresia, L. (2004). Rendimiento de Estudiantes universitarios y sus determinantes. Revista de Economía y Estadística, 42(1) 93-113. https://revistas.unc.edu.ar/index.php/ REyE/article/view/3800

Prado, R., y De Águila, M. (2003). Diferencia en la Resiliencia según género y Nivel Socioeconómico en adolescentes. Persona, 1(6), 179- 196. https://www.redalyc.org/articulo. oa?id=147118110009

Puerta, E., y Vásquez, M. (2012). Caminos para la Resiliencia. Previva.udea, 1(2), 1-3. http:// www.udea.edu.co/wps/wcm/connect/udea/8053e5b2-7d04-4997-9dc0-4dcada70658f/ BoletÃn+2 Concepto+de+Resiliencia.pdf?

Quispe, V., y Vera, L. (2017). Diferencias en la Resiliencia según factores sociodemográficos en Estudiantes de 8 a 12 años de zonas marginales de los Distritos de Cerro Colorado y Cayma [Trabajo de grado, Universidad Nacional de San Agustin, Perú] http://repositorio.unsa.edu. pe/bitstream/handle/UNSA/3540/Psqunuv.pdf?sequence=1\&isAllowed=y

Rabell, M. (octubre de 2012). Autoestima y rendimiento académico: Un estudio aplicado al aula de educación Primaria [Tesis de Maestría, Universidad de la Rioja]. https://reunir.unir. net/bitstream/handle/123456789/1009/2012 1114 TFM ESTUDIO DEL TRABAJO\%20. pdf? sequence $=1$

Rodriguez, H., Guzmán, L., y Yela, N. d. (2012). Factores personales que influyen en el desarrollo de la Resiliencia en niños y niñas en edades entre 7 y 12 años que se desarrollan en extrema pobreza. International Journal of Psychological Research, 5(2), 98-107. http://www.scielo. org.co/pdf/ijpr/v5n2/v5n2a11.pdf 
Rojas, M. J., Arbeláez, D. F., y Prieto, J. D. (2016). Análisis del desempeño escolar de Estudiantes de Secundaria en función de asignaturas, estrato socioeconómico y conflicto armado. Revista amazonia investiga, 6(10), 78 -87. https://www.udla.edu.co/revistas/index.php/amazoniainvestiga/article/download/633/pdf 30

Suleman, Q., Aslam, H. D., Hussain, I., Shakir, M. y Khan, F. U. (2012). Effects of parental socioeconomic status on the academic achievement of secondary schools' students in Karak District, Pakistan. International Journal of Human Resource Studies, 2(4), 14-32.

Tuñón, I. y Poy, S. (2016). Factores asociados a las calificaciones escolares como proxy del rendimiento educativo. Revista Electrónica de Investigación Educativa, 18(1), 98-111. http://redie.uabc. mx/redie/article/view/615

Salgado, A. C. (2005). Métodos e instrumentos para medir la Resiliencia, una alternativa peruana. Liberabit, 11(11), 41-48. http://pepsic.bvsalud.org/scielo.php?pid=S1729$48272005000100006 \&$ script $=$ sci abstract\&tlng $=$ en

Santiago, M., Vergel, M., \& Gallardo, H. (2020). Resiliencia en Estudiantes exitosos en matemáticas. Praxis \& Saber, 11(26), e9973. https://doi.org/10.19053/22160159.v11.n26.2020.9973

Turizo, C. (2018). Informe Municipal de victimas del conflicto. Alcaldía Municipal de Talaigua Nuevo.

Vanistendael, S. (2005, 11 de Noviembre). La Resiliencia: Desde una inspiración hacia los cambios prácticos [Ponencia]. Segundo Congreso internacional de los transtornos en el comportamiento de niños y adolescentes, Madrid, España https://www.yumpu.com/es/ document/read/13238536/stefan-vanistendael-traducida-navarra

Villalta, M. A. (2010). Factores de Resiliencia asociados al rendimiento académico en Estudiantes de contextos de alta vulnerabilidad social. Revista de Pedagogía, 31(88), 159-190. http:// www.scielo.org.ve/scielo.php?pid=S0798-97922010000100007\&script $=$ sci arttext\&tlng $=p t$

Werner, E., y Smith, R. (1992). Overcoming the odds: High risk children. Cornell University Press.

Wolin, S., y Wolin, S. (1993). The resilient self: how survivors of troubled families rise above adversity. Villard Books.

Yapura, M. E. (2015). Estudio sobre la incidencia de la baja Autoestima en el rendimiento academico de los primeros años de escolaridad. [Trabajo de grado, Universidad Abierta Interamericana]. http://imgbiblio.vaneduc.edu.ar/fulltext/files/TC118097.pdf 